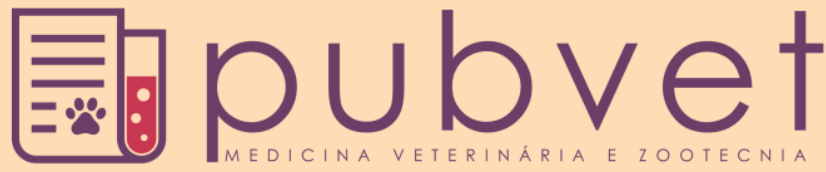

HTTP://DX.DOI.ORG/10.22256/PUBVET.V11N12.1220-1226

\section{Diagnóstico e tratamento do embolismo fibrocartilaginoso em um cão de pequeno porte: Relato de caso}

\author{
Rafaela Macedo Mendes Cozac ${ }^{1 *}$, Fernanda Silva Franco Oliveira ${ }^{2}$, Gizele Monsueth \\ Melo $^{3}$
}

${ }^{1}$ Médico Veterinário, Brasília - DF, Brasil.E-mail: rafamcozac@gmail.com

${ }^{2}$ Graduanda do Curso de Medicina Veterinária das Faculdades ICESP, Brasília - DF, Brasil. E-mail: fernandafrancoo90@gmail.com ${ }^{3}$ Professora Mestre do Curso de Medicina Veterinária das Faculdades ICESP, Brasília - DF, Brasil. E-mail: gizelemonsueth@ gmail.com *Autor para correspondência

RESUMO. A mielopatia por embolismo fibrocartilaginoso é caracterizada por um infarto da medula espinhal causado por êmbolos fibrocartilaginosos idênticos ao núcleo pulposo do disco intervertebral. De ocorrência rara e descrita principalmente em cães adultos e de grande porte, podendo também ser encontrada em cães de pequeno porte. O presente relato ocorreu em um canino de dois anos e meio, macho West Highland White Terrier, que foi atendido em um hospital veterinário com tetraparesia súbita, onde permaneceu internado vinte e um dias e após receber a alta hospitalar deu continuidade ao tratamento por cento e sessenta dias. O diagnóstico foi realizado por meio dos sinais clínicos, exames bioquímicos para exclusão dos diagnósticos diferenciais e a tomografia computadorizada que identificou o trombo em região cervical. O tratamento consistiu em cuidados de enfermagem, fisioterapia, hidroterapia e sessões de acupuntura. Após 180 dias de lesão medular o animal ficou com o membro dianteiro direito sem flexão completa de cotovelo como sequela de uma provável lesão do plexo braquial, com sensibilidade a dor profunda, locomovendo-se com ajuda de um andador e se levantando sozinho.

Palavras chave: cães, embolismo fibrocartilaginoso, isquemia medular

\section{Diagnosis and treatment of fibrocartilaginous embolism in a small dog: Case Report}

\begin{abstract}
Myelopathy due to fibrocartilaginous embolism is characterized by an infarction of the spinal cord caused by fibrocartilaginous embolism identical to the nucleus pulposus of the intervertebral disc. Of rare occurrence and described mainly in adult and large dogs, it can also be found in small dogs. The present report occurred in a two and a half year old canine, West Highland White Terrier, who was treated at a veterinary hospital with sudden tetraparesis, where he remained hospitalized for twenty one days and after being discharged, treatment for sixty days. The diagnosis was made through clinical signs, biochemical tests to exclude differential diagnoses and computed tomography that identified the thrombus in the cervical region. The treatment consisted of nursing care, physiotherapy, hydrotherapy and acupuncture sessions. After 180 days of spinal cord injury, the animal had the right front limb without full elbow flexion as a sequel of a probable brachial plexus injury, with sensitivity to deep pain, walkking with the aid of a walker and rising alone.
\end{abstract}

Keywords: Dogs, fibrocartilaginous embolism, medullar ischemia 


\section{Diagnóstico y tratamiento del embolismo fibrocartilaginoso en perro de pequeño porte: Relato de caso}

RESUMEN. La mielopatía por embolismo fibrocartilaginoso se caracteriza por un infarto de la médula espinal causado por émbolos fibrocartilaginosos idénticos al núcleo pulposo del disco intervertebral. De ocurrencia rara y descrita principalmente en perros adultos y de gran porte, pudiendo también ser encontrada en perros pequeños. El presente relato se produjo en un canino de dos años y medio, macho West Highland White Terrier, que fue atendido en un hospital veterinario con tetraparesia súbita, donde permaneció internado veintiún días y tras recibir el alta hospitalaria dio continuidad al tratamiento por ciento y Sesenta días. El diagnóstico fue realizado por medio de los signos clínicos, exámenes bioquímicos para la exclusión de los diagnósticos diferenciales y la tomografía computarizada que identificó el trombo en región cervical. El tratamiento consistió en cuidados de enfermería, fisioterapia, hidroterapia y sesiones de acupuntura. Después de 180 días de lesión medular el animal se quedó con el miembro delantero derecho sin flexión completa de codo como secuela de una probable lesión del plexo braquial, con sensibilidad al dolor profundo, moviéndose con ayuda de un andador y levantándose solo.

Palabras clave: Embolismo fibrocartilaginoso, isquemia medular, perros

\section{Introdução}

A mielopatia por embolismo fibrocartilaginoso é caracterizada por um infarto da medula espinhal ocasionada por êmbolos de fibrocartilagem proveniente do núcleo pulposo dos discos intervertebrais (Nelson and Couto, 2015). Sua ocorrência é rara e tem sido descrita em cães, gatos, suínos, equinos, bovinos, tigres, cordeiros e humanos. Em cães, esta doença acomete principalmente animais adultos e de grande porte; contudo, assim como no presente caso, também tem sido encontrada em cães de raças de pequeno e médio porte com freqência relativa na raça schnauzer e em cães condrodistróficos. Quanto a fisiopatologia do embolismo fibrocartilaginoso (EFC) ainda é incerto como o êmbolo histologicamente compatível com o núcleo pulposo do disco intervertebral chega nos vasos da medula espinhal (De Risio and Platt, 2010, De Risio, 2015, Garavito-Bernal and León-Torres, 2015). As seguintes terorias são propostas:

- Com o aumento da pressão intraabdominal e intratorácica durante um pequeno trauma como atividade física, pode ocorrer penetração direta de fragmentos do núcleo pulposo por meio de propulsão venosa retrógada do êmbolo nas artérias e veias espinhais intrínsecas. É relatado com maior frequência em cães (De Risio and Platt, 2010, De Risio, 2015, Garavito-Bernal and León-Torres, 2015, De Risio, 2014).

- Hérnia do núcleo pulposo com exposição da vasculatura do corpo vertebral, por meio de manipulação direta durante procedimentos cirúrgicos ou traumatismos dentro da porção esponjosa do corpo cervical, e se observa somente em humanos (De Risio and Platt, 2010, De Risio 2015, Garavito-Bernal and León-Torres, 2015).

- Neovascularização inflamatória crônica do disco intervertebral degenerado. Um aumento repentino da pressão intradiscal que exceda a pressão arterial pode facilitar a penetração de fibrocartilagem do núcleo pulposo dentro dos vasos sanguíneos do disco degenerado, chegando à medula espinhal (De Risio and Platt, 2010, De Risio, 2015, Garavito-Bernal and León-Torres, 2015).

- Persistência de vasos remanescentes embrionários dentro do núcleo pulposo (De Risio and Platt, 2010, De Risio, 2015, Garavito-Bernal and León-Torres, 2015, De Risio, 2014).

- Desenvolvimento de uma metaplasia fibróide do núcleo pulposo, onde ocorre a perda da viscoelasticidade do anel intervertebral em cães adultos e condrodistróficos com possível ruptura parcial do anel fibroso e uma neovascularização que comunica o núcleo pulposo com a artéria espinhal ou artérias radiculares (De Risio and Platt, 2010, De Risio, 2015, Garavito-Bernal and León-Torres, 2015).

- Outra hipótese sugere que a fibrocartilagem sai desde a placa de crescimento da cartilagem vertebral de cães jovens e como conseqüência ocasiona a embolização dentro da vasculatura intrínseca da medula espinhal (De 
$\underline{\text { Risio and Platt, 2010, De Risio, 2015, Garavito- }}$ Bernal and León-Torres, 2015).

Independente do mecanismo, a oclusão com um êmbolo fibrocartilaginoso em uma artéria ou veia resulta em uma isquemia local, que afeta todo o metabolismo aeróbio, gerando alterações bioquímicas, eletrolíticas e vasculares que ocasionam perda da polaridade nas membranas celulares seguido de uma cascata de eventos destrutivos como apoptose (De Risio and Platt, 2010, De Risio, 2015, Garavito-Bernal and LeónTorres, 2015, De Risio, 2014).

A história clínica, os sinais clínicos, a realização de exames neurológicos e a utilização de ferramentas diagnósticas como radiografias, mielografia, ressonância magnética, tomografia computadorizada (TC) e outros exames complementares permitem uma maior precisão diagnóstica. Ainda, a exclusão de outras mielopatias. Entretanto, o diagnóstico definitivo do EFC só pode ser concluído post mortem por meio do exame neurohistológico (De Risio and Platt, 2010, De Risio, 2015, Garavito-Bernal and León-Torres, 2015, De Risio, 2014).

O tratamento do EFC consiste em cuidados de enfermagem, reabilitação com fisioterapia, terapêutica medicamentosa com antiinflamatórios esteróides, substâncias antioxidantes e neuroprotetores (De Risio and Platt, 2010, Mateen et al., 2011, De Risio, 2015, Garavito-Bernal and León-Torres, 2015, De Risio, $\underline{2014) \text {. }}$

A localização da lesão causada pelo EFC influência diretamente na recuperação $\mathrm{e}$ prognóstico do paciente. A presença de nocicepção e sensibilidade a dor profunda são indicativos de um bom prognóstico; enquanto a ausência de dor profunda sugere um prognóstico reservado (De Risio and Platt, 2010, De Risio, 2015, Garavito-Bernal and León-Torres, 2015, De Risio, 2014).

\section{Material e Métodos}

Foi atendido em um hospital veterinário de Brasília - DF, um canino da raça West Highland White Terrier, macho, 2 anos e meio de idade com peso de $8,5 \mathrm{~kg}$.

O paciente encontrava-se em decúbito lateral, normocorado, hidratado, normotérmico, taquipnéico, com membros torácicos e pélvicos ligeiramente espásticos, reflexo fotopupilar direto e consensual presente e consciência ligeiramente diminuída. O proprietário relatou que o animal passou o dia bem, comeu, bebeu água, defecou e urinou normalmente, mas que acordou de madrugada com o animal chorando, estendido no chão, se debatendo muito e que pensou tratar-se de um quadro convulsivo. Foi levado rapidamente para uma clínica mais perto da sua residência, onde recebeu o primeiro atendimento do médico veterinário plantonista, que realizou hemograma completo e fez medicação com fenobarbital e dipirona sódica $\mathrm{EV}$, com dosagem desconhecida pelo proprietário. $\mathrm{O}$ hemograma não revelou quaisquer alterações. O proprietário nega quadros convulsivos anteriores e traumas. Todavia, afirma que o animal é bastante ativo, gosta de correr e não possui qualquer problema de saúde pregresso e que poderia ter tido contato com animais mortos, uma vez que passou muito tempo na área externa onde seria de costume ser encontrado dejetos de animais em decomposição. $\mathrm{O}$ paciente seguiu internado para realização de avaliação com a neurologia e exames complementares. Ao segundo dia de internação o animal passou por avaliação com a neurologia, onde foi constatado que não havia movimentação voluntária do pescoço, mas sustentava a cabeça quando em esternal, os reflexos de nervos cranianos encontraram-se normais, sem reflexos aos estímulos dolorosos e com tetraparesia flácida. As principais suspeitas diagnósticas seriam botulismo, mielite, meningite e embolismo fibrocartilaginoso (EFC).

\section{Resultados}

Prescreveu-se prednisona $0,5 \mathrm{mg} / \mathrm{kg}$ BID e tramadol $4 \mathrm{mg} / \mathrm{kg}$ BID EV. O animal comeu espontaneamente, bebeu água, defecou, e urinou via sonda vesical. Ao terceiro dia de internação o paciente foi submetido à tomografia de coluna cervical que evidenciou isquemia medular em região $\mathrm{C} 6$ sugestivo de embolismo fibrocartilaginoso (EFC), RX de coluna cervical normal, EAS compatível com cistite e demais exames bioquímicos dentro da normalidade. Foi acrescentado a prescrição médica enrofloxacino 5 $\mathrm{mg} / \mathrm{Kg}$ BID e Polivin B12, $2 \mathrm{~mL}$, SID. Paciente seguiu com cuidados de enfermagem e medicações. No quinto dia de internação a prednisona e o tramadol foram suspensos e o paciente seguiu com cuidados de enfermagem e antibioticoterapia e permaneceu com a sonda vesical.

Ao sexto dia foram iniciadas as sessões de fisioterapia e aplicação de ozonioterapia onde foi 
observada melhora significativa do reflexo de retirada e reflexos aos estímulos dolorosos presentes, já com sustentação de membros pélvicos quando colocado em estação. Ao décimo quarto dia de internação, o paciente apresentou quadro de hipertermia. Foi realizado o hemograma que apresentou leucocitose, e trombocitopenia, RX tórax normal, EAS compatível com cistite, US abdominal com parede da bexiga mais evidente, foi aumentada a dose do enrofloxacino para 10 $\mathrm{mg} / \mathrm{kg}$ BID e a sonda vesical foi retirada. Paciente urinou sozinho e por compressão da bexiga nos dias posteriores. No décimo sexto dia houve um aumento significativo da leucocitose e a antibióticoterapia foi modificada e introduziu-se meropenem $24 \mathrm{mg} / \mathrm{kg}$ EV. Paciente seguiu com cuidados de enfermagem, fisioterapia, medicações e monitoração da infecção urinária. Ao vigésimo primeiro dia de internação o paciente recebeu alta, com hemograma e demais exames normais, alimentando-se bem, bebendo água, defecando normalmente e urinando espontaneamente. No entanto, por vezes com compressão da bexiga, ainda com tetraparesia e com prescrição de prazosina $0,4 \mathrm{ml}$ BID e suplementação com vitamina E 60UI BID e sessões de fisioterapia duas vezes por semana e acupuntura uma vez por semana. A prazosina foi suspensa 15 dias após a alta hospitalar e o proprietário relatou que o animal passou bem e sem alterações durante o período de alta. Após 50 dias de lesão o animal já conseguia levantar-se por vezes sozinho, mas ainda não tinha sustentação para permanecer em estação. As sessões de fisioterapia consistiram em hidroterapia, magnetoterapia, ozonioterapia alternadamente. $\mathrm{O}$ animal seguiu com as sessões de fisioterapia e acupuntura e após seis meses de lesão passou a locomover-se com andador, onde houve um discreto aumento do tônus muscular e da força motora. O membro torácico direito permaneceu sem flexão de cotovelo como sequela, mas com sensibilidade a dor profunda.

\section{Discussão}

O diagnóstico do EFC é realizado mediante a histórica clínica, sinais clínicos, realização do exame neurológico completo e outras ferramentas diagnósticas como RX, mielografia, ressonância magnética e tomografia computadorizada. Entretanto, o diagnóstico definitivo e o mais preciso é o exame histológico post mortem com coleta do segmento da lesão medular e posterior coloração e análise microscópica (Nakamoto et al., 2009, De Risio and Platt, 2010, Mateen et al.,
2011, Chaves et al., 2013, AbdelRazek et al., 2016, De Risio, 2015, Garavito-Bernal and LeónTorres, 2015, Chaves et al., 2016).

O RX permite descartar fraturas, luxações, neoplasias, e enfermidades intervertebrais. No $\mathrm{EFC}$ as radiografias normalmente não apresentam quaisquer alterações. A mielografia possui grande valor diagnóstico para exclusão de outras causas de mielopatia aguda compressiva, normalmente no quadro de EFC pode sugerir uma inflamação focal intramedular no período agudo. A Tomografia Computadorizada (TC) também pode ajudar na exclusão de outras mielopatias compressivas, na fase aguda pode apresentar edema focal medular, demonstrada com regiões de hipodensidade. A ressonância magnética é o método diagnóstico mais preciso in vivo na medicina veterinária e humana, as principais alterações observadas em pacientes com EFC são lesões intramedulares focais, assimétricas, bem delimitadas com regiões hiperintensaso (De Risio, 2015, Garavito-Bernal and León-Torres, 2015).

O diagnóstico do caso clínico foi baseado pela história clínica, sinais clínicos apresentados pelo cão e resultado da Tomografia Computadorizada (Figura 1). O animal iniciou subitamente com os sintomas, não houve relatos de traumas físicos, nem história pregressa que poderia indicar outras patologias. Além disso, apresentou todos os exames, como RX, hemograma e perfil bioquímico, normais o que levaria a exclusão de alguns diagnósticos diferenciais. $\mathrm{O}$ botulismo seria uma das possibilidades de diagnóstico diferencial no caso, pois o animal poderia ter tido contato com animais em processo de decomposição, conforme constatado na anamnese. Todavia o exame de pesquisa da toxina botulínica apresentou resultado negativo. A TC foi realizada após o terceiro dia de lesão, ou seja, na fase aguda do curso da doença. Neste caso o laudo médico sugere diagnóstico compatível com EFC, devido à lesão de isquemia medular em região C6 que apresentava região de hipodensidade na mesma área, mas que o diagnóstico definitivo só poderia ser feito por meio da ressonância magnética. Embora a TC não seja o método mais preciso de diagnóstico, ela exclui algumas mielopatias de compressão medular, como a extrusão do disco cervical e traumas (Nakamoto et al., 2009, De Risio and Platt, 2010, Mateen et al., 2011, Chaves et al., 2013, AbdelRazek et al., 2016, De Risio, 2015, Garavito-Bernal and León-Torres, 2015, Chaves et al., 2016). Haja vista que o animal realizou o exame na fase aguda, onde este é mais 
sensível e a região de hipodensidade indica fortemente a presença de edema na região, compatível com as lesões encontradas em TC com EFC (De Risio and Platt, 2010, Mateen et al., 2011, De Risio, 2015, Garavito-Bernal and León-Torres, 2015, De Risio, 2014).

As melhoras progressivas da sintomatologia neurológica também foram de suma importância para fechar o diagnóstico. Não foi realizada mielografia e análise do líquido cefalorraquiano. A ressonância magnética não pode ser feita devido a não existir aparelho veterinário na localidade.

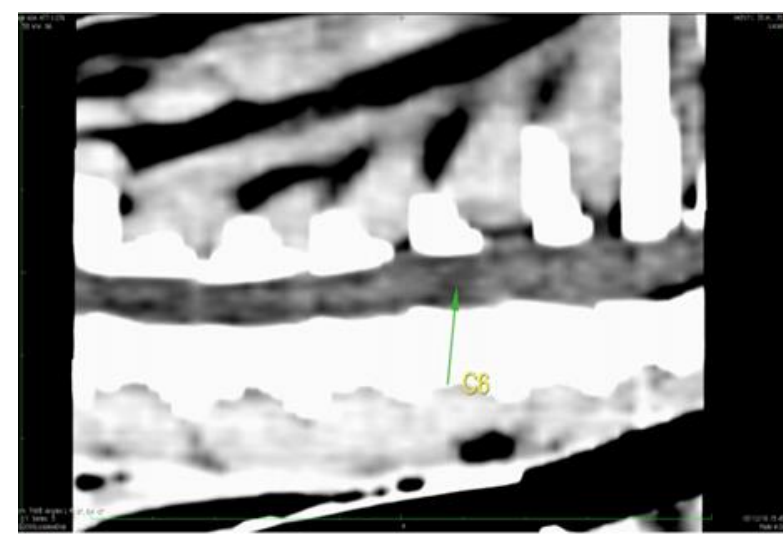

Figura 1. Tomografia Computadorizada (TC) com lesão evidente em região C6.

Quanto ao tratamento do EFC os principais objetivos são: reduzir as possíveis lesões medulares, manter a perfusão da medula espinhal e a neuroproteção. Os agentes anti-inflamatórios corticoides como o succinato sódico de metilprednisona é mais efetivo quando iniciado dentro das primeiras 8 após o surgimento dos sinais clínicos, com dose de $30 \mathrm{mg} / \mathrm{kg}$ lentamente por quinze minutos, seguido de uma pausa de 45 minutos e infusão contínua de $5.4 \mathrm{mg} / \mathrm{kg} / \mathrm{hora}$, durante 23 horas. Desta forma pode se diminuir o edema pós-traumático e atua como neuroprotetor. $\mathrm{O}$ uso da pentoxifilina na dosagem de $10 \mathrm{mg} / \mathrm{kg}$ via oral, parece evitar o progresso do dano medular, mas deve ser administrada após a implementação da terapia anti-inflamatória, neuroprotetora e antioxidante. A vitamina $\mathrm{E}$ deve ser administrada a uma dose de 400 UI a cada 24 horas para ter uma ação antioxidante com ação de eliminação dos radicais livres e consequente proteção das membranas celulares. Além da terapia farmacológica, se recomenda a fisioterapia com hidroterapia, massagens, movimentos passivos, eletroterapia e cuidados gerais de enfermagem (Tesser et al., 2009, De Risio and Platt, 2010, De Risio, 2015, Garavito-Bernal and
León-Torres, 2015, De Risio, 2014).

No caso clínico relatado, o animal fez uso da prednisona somente após 20 horas do surgimento dos sintomas, e em uma dosagem bem abaixo do recomendado, o que talvez tenha contribuído para a permanência do edema medular e consequente atraso na recuperação. A pentoxifilina não foi utilizada no protocolo terapêutico devido ao pouco conhecimento da sua utilização associado ao EFC. A vitamina $E$ foi suplementada após a alta hospitalar em dosagem bem abaixo do recomendado, porém nas primeiras 24 horas após o surgimento dos sinais clínicos, o animal foi suplementado com vitamina B12 a fim de se obter um resultado neuroprotetor. São conhecidos os efeitos neuroprotetores da vitamina B12 (Paniz et al., 2005). Aos primeiros 15 dias de internação, o animal não urinava espontaneamente e fez uso da prasozina e compressão manual para promover o relaxamento vesical e após a alta hospitalar já conseguia urinar sozinho e a medicação foi suspensa. Nesses casos, é recomendado o uso de diazempam com dose de $0,25 \mathrm{mg} / \mathrm{kg}$ e também a compressão manual para evitar o dano vesical por excessiva distensão e distúrbios eletrolíticos. A prasozina obteve um efeito satisfatório na melhora da retenção urinária (De Risio and Platt, 2010, Garavito-Bernal and León-Torres, 2015).

$\mathrm{O}$ animal iniciou o tratamento fisioterápico logo nos primeiros dias após a lesão, com melhora progressiva dos movimentos, tônus muscular, reflexos e sustentação. No tratamento inicial, ainda durante a hospitalização a fisioterapia era movimentação passiva, massagens e ozonioterapia. Após a alta o animal passou a realizar sessões de hidroterapia, eletroterapia, magnetoterapia e movimentação mais ativa, uma vez que já conseguia manter certa sustentação. Realizou concomitantemente sessões de acupuntura e eletroacupuntura uma vez por semana, onde obteve um grande progresso na região motora e sensitiva. Após 6 meses de lesão o animal não se recuperou completamente, embora por vezes consiga levantar-se sozinho. Possui sensibilidade a dor profunda nos membros torácicos e pélvicos, e caminha com ajuda do andador. $\mathrm{O}$ membro torácico direito permaneceu sem o arco reflexo, mas com sensibilidade a dor profunda e o membro pélvico esquerdo com uma hiper-reflexia ao tocar as superfícies, com melhora progressiva após o uso do andador.

$\mathrm{O}$ tratamento com células tronco em um cão com EFC devido a extrusão do disco intervertebral 
obteve resultados satisfatórios nos reflexos espinhais e nas funções locomotoras, mas não restabeleceu a sensibilidade a dor profunda do animal após um ano do tratamento. Um mau prognóstico do EFC é a ausência de dor profunda e reflexos espinhais (Chung et al., 2013).

Parece que a localização da lesão do EFC não influencia na recuperação dos pacientes, entretanto alguns estudos demonstram que o tempo de recuperação parece ser influenciado pela a localização da lesão. Em pacientes com lesões neuromotoras superiores (T3-L3 e C1-C5) se recuperam, mais rápido do que com lesões neuromotoras inferiores. A recuperação neurológica na maioria dos estudos ocorre dentro de 10 a 45 dias, mas pode variar de conforme a extensão da lesão. Cuidados de enfermagem e fisioterapia devem ser continuados pelo menos 2 meses após o surgimento dos sintomas clínicos (Garavito-Bernal and León-Torres, 2015, Chaves et al., 2016).

A lesão do animal foi em região C6, ou seja, mais caudal à região cervical, o que talvez seja a causa da lenta recuperação do paciente, que apesar de ter tido uma grande melhora clínica, ainda não obteve completa recuperação, mesmo com a fisioterapia e estímulos intensificados. A demora na administração do anti-inflamatório também pode ter contribuído para que o edema local aumentasse a lesão medular infartada assim como a não utilização da pentoxifilina e da vitamina $\mathrm{E}$ pode ter contribuído com o progresso do dano medular.

\section{Considerações Finais}

É importante ressaltar que a baixa incidência do EFC, principalmente em pequenos animais é um fator limitante no sucesso do tratamento, pois ainda existem poucos casos relatados e pesquisas acerca do tratamento. Contudo mais estudos seriam necessários para a criação de um protocolo de tratamento, principalmente nas primeiras horas de lesão do EFC para que as chances de melhora total e de um bom prognóstico sejam eficientes.

\section{Referências Bibliográficas}

AbdelRazek, M. A., Mowla, A., Farooq, S., Silvestri, N., Sawyer, R. \& Wolfe, G. 2016. Fibrocartilaginous embolism: a comprehensive review of an under-studied cause of spinal cord infarction and proposed diagnostic criteria. The Journal of Spinal Cord Medicine, 39, 146-154.
Chaves, R. O., Beckmann, D. V., Santos, R. P., Andrades, A. O., Macagnan, M., Aiello, G., Soares, A. V. \& Mazzanti, A. 2013. Diagnóstico presuntivo de embolismo fi brocartilaginoso em 12 cães. Acta Scientiae Veterinariae, 41, 1-5.

Chaves, R. O., Copat, B., Beckmann, D. V., Feranti, J. P. S., Oliveira, M. T., Corrêa, L. F. D., Ripplinger, A. \& Mazzanti, A. 2016. Embolismo fibrocartilaginoso associado à extrusão do disco intervertebral em cão. Acta Scientiae Veterinariae, 44, 1-4.

Chung, W.-H., Park, S.-A., Lee, J.-H., Chung, D.J., Yang, W.-J., Kang, E.-H., Choi, C.-B., Chang, H.-S., Kim, D.-H. \& Hwang, S.-H. 2013. Percutaneous transplantation of human umbilical cord-derived mesenchymal stem cells in a dog suspected to have fibrocartilaginous embolic myelopathy. Journal of Veterinary Science, 14, 495-497.

De Risio, L. 2014. What is fibrocartilaginous embolism and is it related to IVDD? In: M.F., J. \& B.T., W. (eds.) Advances in intervertebral disc disease in dogs and cats.

De Risio, L. 2015. A review of fibrocartilaginous embolic myelopathy and different types of peracute non-compressive intervertebral disk extrusions in dogs and cats. Frontiers in Veterinary Science, 2, 1-9.

De Risio, L. \& Platt, S. R. 2010. Fibrocartilaginous embolic myelopathy in small animals. Veterinary Clinics of North America: Small Animal Practice, 40, 859-869.

Garavito-Bernal, N. \& León-Torres, W. A. 2015. Embolismo fibrocartilaginoso de la medula espinal en caninos. Zoociencia, 2, 12-19.

Mateen, F. J., Monrad, P. A., Leep Hunderfund, A. N., Robertson, C. E. \& Sorenson, E. J. 2011. Clinically suspected fibrocartilaginous embolism: clinical characteristics, treatments, and outcomes. European Journal of Neurology, 18, 218-225.

Nakamoto, Y., Ozawa, T., Katakabe, K., Nishiya, K., Yasuda, N., Mashita, T., Morita, Y. \& Nakaichi, M. 2009. Fibrocartilaginous embolism of the spinal cord diagnosed by characteristic clinical findings and magnetic resonance imaging in 26 dogs. Journal of Veterinary Medical Science, 71, 171-176.

Nelson, R. W. \& Couto, C. G. 2015. Medicina interna de pequenos animais. Elsevier Editora, Amsterdan. 
Paniz, C., Grotto, D., Schmitt, G. C., Valentini, J., Schott, K. L., Pomblum, V. J. \& Garcia, S. C. 2005. Fisiopatologia da deficiência de vitamina B12 e seu diagnóstico laboratorial. Jornal Brasileiro de Patologia Médica e Laboratorial, 41, 323-34.

Tesser, E. S., Pavarini, S. P., Bezerra Junior, P. S., Marcato, J. d. A., Antoniassi, N. A. B., Voll, R. \& Driemeier, D. 2009. Mielopatia por embolismo fibrocartilaginoso em um canino. Acta Scientiae Veterinariae, 37, 291-295.

\section{Article History:}

Received 24 July 2017

Accepted 18 August 2017

Available on line 18 October 2017

License information: This is an open-access article distributed under the terms of the Creative Commons Attribution License 4.0, which permits unrestricted use, distribution, and reproduction in any medium, provided the original work is properly cited. 